\title{
Health-Related Problems after the Great East Japan Earthquake: An Evaluation Based on the Annual Health Examination
}

\author{
${ }^{1}$ Health and Global Policy Institute, Japan \\ ${ }^{2}$ Graduate School of Medicine, the University of Tokyo, Japan \\ ${ }^{3}$ Institute of Industrial Ecological Sciences, University of Occupational and Environmental Health, Japan \\ ${ }^{4}$ National Cancer Center, Japan \\ ${ }^{5}$ School of Nursing, National College of Nursing, Japan \\ ${ }^{6}$ Office of a member of the House of Councilors, Japan \\ ${ }^{7}$ Carepro Co., Ltd., Japan
}

Kazumi Kubota ${ }^{1,2^{*}}$, Akiomi Inoue ${ }^{3}$, Yoichi Shimizu ${ }^{4}$, Satoko Kagata ${ }^{5}$, Roseline Yong ${ }^{2}$, Yoshiaki Hirama $^{6}$, Masaru Shiga ${ }^{7}$, Takashi Kawazoe ${ }^{7}$

\begin{abstract}
Background: Study on the medium- to long-term effect of a great earthquake on health among the victims is limited. The purpose of the present study was to investigate the medium-term (i.e., three months) effect of the Great East Japan Earthquake (occurred on March 11, 2011) on health conditions (i.e., blood pressure, hemoglobin A1c [HbA1c], blood sugar, triglyceride, and total cholesterol) among the victims, by comparing with the control population.

Methods: In June 2011, taking blood pressure and blood tests were conducted for a total of 159 victims who had stayed in the shelter located in the Tohoku region since the Great East Japan Earthquake. The same tests were conducted for a total of 1,048 control participants in Tokyo from October 2010 to December 2011. Analyses of covariance and multiple logistic regression analyses were conducted.

Results: Compared with the control group, the victim group had significantly higher systolic and diastolic blood pressure; lower $\mathrm{HbA} 1 \mathrm{c}$, blood sugar, triglyceride, and total cholesterol, after adjusting for age and sex. In a similar way, the victim group had a significantly higher prevalence odds ratio of hypertension; lower prevalence odds ratios of diabetes mellitus, high blood sugar, high triglyceride, and high total cholesterol than control group.

Conclusions: The present study revealed that the prevalence of hypertension is higher among the victims of the Great East Japan Earthquake. Lower prevalence of diabetes mellitus, high blood sugar, high triglyceride, and high total cholesterol among the victims may be explained by the continued inadequate diet issues after the earthquake.
\end{abstract}

Keywords: Health-related problems; The great east Japan earthquake; Annual health examination; Analyses of covariance; Multiple logistic regression analyses

\section{Introduction}

A magnitude 9.0 earthquake occurred on March 11, 2011, 14:46 at the epicenter about $130 \mathrm{~km}$ off the coast of Sanriku Northeast. The earthquake caused an onslaught of tsunami up to $15 \mathrm{~m}$ or more, had significant damage the coastal areas in the Tohoku region. After nine months of the tsunami, 15,842 deaths were reported, 3,481 missing, and about 18,000 people were spending their lives in the shelter [1]. Majority of those who lived in the shelter would end up living in the temporary housing later, where the huge environment and lifestyle changes are predicted to cause a great distress to the victims. The stress of the victims are not only limited to the immediate disaster stress response, but also after disaster stress response, which is chronic, and is predicted to leave an impact towards psychological and physical health. The death of stroke and cardiovascular events at one municipality were reported to be doubled than the previous year after three months of the Great Hanshin-Awaji Earthquake in 1995 [2]. Therefore it is thought that while considering the health issues of the disaster victims, the management of hypertension and dyslipidemia is essentially important as these are the major risk factors for cardiovascular events.

Other reports in Japan had also revealed that the raise of the blood pressure is noticed directly after disaster, as well as few weeks after disaster [2-5]. In the Great Hanshin-Awaji Earthquake event, among the 221 hypertensive patients who were under medical treatment, the blood pressure and heart rate were assessed with the same treatment before disaster, and it was found that there was neither significant change of blood pressure nor heart rate change in the non-disaster group before and after the disaster. However in the disaster group, significant rise in blood pressure and heart rate was found four weeks after the disaster [3]. The other studies reported that the victims with raised blood pressure after Great Hanshin-Awaji Earthquake tended to resume to a normal blood pressure level after four weeks $[4,5]$, these observations suggest that the acute rise of blood pressure in the victims is recovered after few weeks. However it was also pointed out that the hypertension rate may increase after a few years following the disaster [2-5], therefore a medium- to long-term observation is necessary. The health checks data before and after the Chuetsu Earthquake in 2004 showed that dyslipidemia in the Niigata people was significant rose after the event [6]. It was pointed out as food is normally insufficient in the disaster areas, the imbalance of nutrition may result in hypoglycemia [7]. In a study of the Type 2 diabetes patients of the Chuetsu Earthquake victims, glycemic control after event is significantly worse than before [8].

By learning from the previous health studies of disasters [2-8], and by looking at the difficulties in health care management accompanied with the huge change of living environment seen in the Great East Japan Earthquake, it is predicted that the health conditions of the victims

${ }^{*}$ Corresponding author: Kazumi Kubota, Department of Mental Health, Graduate School of Medicine, The University of Tokyo, 7-3-1 Hongo, Bunkyo-ku, Tokyo 113-0033, Japan, Tel: +81-(0)3-5841-3522; Fax: +81-(0)3-5841-3392; E-mail: tothankyouall@gmail.com

Received April 23, 2013; Accepted September 30, 2013; Published October 04 2013

Citation: Kubota K, Inoue A, Shimizu Y, Kagata S, Yong R, et al. (2013) HealthRelated Problems after the Great East Japan Earthquake: An Evaluation Based on the Annual Health Examination. J Nurs Care 2: 134. doi:10.4172/21671168.1000134

Copyright: $\odot 2013$ Kubota K, et al. This is an open-access article distributed under the terms of the Creative Commons Attribution License, which permits unrestricted use, distribution, and reproduction in any medium, provided the original author and source are credited. 
Citation: Kubota K, Inoue A, Shimizu Y, Kagata S, Yong R, et al. (2013) Health-Related Problems after the Great East Japan Earthquake: An Evaluation Based on the Annual Health Examination. J Nurs Care 2: 134. doi:10.4172/2167-1168.1000134

Page 2 of 5

would change as health care is difficult to manage. As in present, the health condition of the victims in the Great East Japan Earthquake has not been few reported, where the situation is difficult to grasp. Previous study for 142 hypertensive outpatients lived in the affected area had shown that, average home systolic BP, but not diastolic BP, was significantly elevated immediately and 2 weeks after, but disappeared 4 weeks after the earthquake when compared with their data collected shortly before the earthquake, [9]. Also, Konno et al. [10] revealed that the systolic and diastolic blood pressure of the public employees increased higher than the general population while compared to the measurements took in the previous year. However, these studies were focused on hypertension, and none of it had investigated the diabetes mellitus and hyperlipidemia with cardiovascular diseases index. Therefore, this study aimed to investigate the health condition among the Great East Japan Earthquake victims, by comparing the health checks data with the non-disaster population. In the present study, because we focused mainly on the effect of life in the shelter on the health condition among the victims, control group was collected from Tokyo residents, who had not lived in the shelter but experienced the consequences of disaster such as series of strong aftershocks, fear of radioactive exposures, and planned blackouts.

\section{Materials and Methods \\ Participants}

In this study, a total of 159 participants (male 69, female 90) that had stayed in the shelter for three months from an affected area (victims) were compared with a total of 1,048 participants (male 558, female 490) from a non-affected area (controls). Inclusion criteria of the victim group were residents (20 age or over) in one of the main disaster area, Miyagi prefecture, in the Tohoku region (Northern East part of Japan), which were forced to reside in the shelters and temporary housings as residential were collapsed due to the earthquake and tsunami. The health data in affected area was collected by 1-3 nurses and non-medical volunteer staffs' team from a health check up company based in Tokyo in June 2011. The nurses carried out the blood test and the volunteer staff was assistants to the nurses. The data of the control group was also collected by the same company's staff using same medical devises (from October 2010 to December 2011). Inclusion criteria of the control group were all above age 20. Informed consents were obtained from both groups of samples, and the participation was voluntarily. The patients were also allowed to decline the study at any time.

Table 1: Demographic characteristics of respondents ${ }^{1)}$

\begin{tabular}{|c|c|c|c|c|c|c|c|c|c|c|}
\hline & \multicolumn{4}{|c|}{ Victim Group } & \multicolumn{4}{|c|}{ Non-victim Group } & \multirow{2}{*}{ Statistical test } & \multirow{2}{*}{$p$ value } \\
\hline & $\mathrm{n}$ & $(\%)$ & Mean & $\mathrm{SD}^{2)}$ & $\mathrm{n}$ & $(\%)$ & Mean & $\mathrm{SD}^{2)}$ & & \\
\hline Age & 159 & & 58.2 & $(15.3)$ & 1048 & & 44.4 & $(14.0)$ & $F(1,1204)=132.00$ & $<0.001$ \\
\hline Sex & & & & & & & & & $x 2(1)=5.36$ & 0.022 \\
\hline Men & 69 & $(43.4)$ & & & 558 & $(53.2)$ & & & & \\
\hline Women & 90 & $(56.6)$ & & & 490 & $(46.8)$ & & & & \\
\hline Systolic blood pressure $(\mathrm{mmHg})$ & 158 & & 141.1 & $(22.5)$ & 495 & & 125.2 & $(19.2)$ & $F(1,651)=75.925$ & $<0.001$ \\
\hline Diastolic blood pressure $(\mathrm{mmHg})$ & 158 & & 87.5 & $(12.4)$ & 495 & & 81.1 & $(12.9)$ & $F(1,651)=29.395$ & $<0.001$ \\
\hline $\mathrm{HbA} 1 \mathrm{c}(\%)$ & 144 & & 5.3 & $(0.6)$ & 244 & & 5.5 & $(1.1)$ & $F(1,386)=3.592$ & 0.059 \\
\hline Blood Sugar (mg/dL) & 158 & & 116.8 & $(37.0)$ & 585 & & 117.2 & $(43.8)$ & $F(1,739)=0.011$ & 0.915 \\
\hline Triglyceride (mg/dL) & 157 & & 153.2 & $(89.8)$ & 660 & & 175.7 & $(121.2)$ & $F(1,815)=4.776$ & 0.029 \\
\hline rota/ cholesterol (mg/dL) & 155 & & 194.6 & $(36.5)$ & 718 & & 203.4 & $07.5)$ & $F(1,871)=7.172$ & 0.008 \\
\hline Hypertension $^{3)}$ & & & & & & & & & $x^{2}(1)=160.91$ & $<0.001$ \\
\hline Yes & 92 & $(42.1)$ & & & 152 & $(14.5)$ & & & & \\
\hline No & 67 & $(57.9)$ & & & 896 & $(85.5)$ & & & & \\
\hline Diabetes $^{3)}$ & & & & & & & & & $x 2(1)=1.48$ & 225 \\
\hline Yes & 13 & $(9.0)$ & & & 32 & $(13.1)$ & & & & \\
\hline No & 131 & $(91.0)$ & & & 212 & $(86.9)$ & & & & \\
\hline High blood sugar ${ }^{3}$ & & & & & & & & & $x^{2}(1)=0.15$ & 0.701 \\
\hline Yes & 40 & $(25.3)$ & & & 139 & $(23.8)$ & & & & \\
\hline No & 118 & $(74.7)$ & & & 444 & $(76.2)$ & & & & \\
\hline High triglyceride $^{3)}$ & & & & & & & & & $x^{2}(1)=2.32$ & 0.128 \\
\hline Yes & 62 & $(39.5)$ & & & 305 & $(46.2)$ & & & & \\
\hline No & 95 & $(60.5)$ & & & 355 & $(53.8)$ & & & & \\
\hline High total cholestero| ${ }^{3)}$ & & & & & & & & & $x^{2}(1)=3.26$ & 0.080 \\
\hline Yes & 36 & $(23.2)$ & & & 219 & $(30.5)$ & & & & \\
\hline No & 119 & $(76.8)$ & & & 499 & $(69.5)$ & & & & \\
\hline
\end{tabular}

1) $p$ values were calculated by analysis of variance (A NOVA) or Chi-square test.

2) SD: Standard deviation.

3) Hypertension, high blood sugar, diabetes, high triglyceride, and high total cholesterol were defined as follows:

Hypertension $=$ Systolic blood pressure $>140 \mathrm{mmHg}$ or Diastolic blood pressure $>90 \mathrm{mmHg}$; Diabetes $=\mathrm{HbAlc}>6.1 \%$;

High blood sugar = Blood sugar $>126 \mathrm{mg} / \mathrm{dL} ;$ High triglyceride $=$ Triglyceride $>150 \mathrm{mg} / \mathrm{dL} ;$ High total cholesterol $=$ Total cholesterol $>220 \mathrm{mg} / \mathrm{dL}$ 


\section{Outcome variables}

Blood pressure and blood test were conducted during the survey. Hemoglobin Alc (HbAlc) (\%), blood sugar (mg/dL), triglyceride (mg/ $\mathrm{dL})$, and total cholesterol $(\mathrm{mg} / \mathrm{dL})$ were measured in the blood test.

\section{Statistical analysis}

Analyses of covariance (ANCOVA) were conducted to compare adjusted systolic and diastolic blood pressure, $\mathrm{HbA1c}$, blood sugar, triglyceride, and total cholesterol between the victim and control groups. In addition, multiple logistic regression analyses were performed to examine whether there were differences in the prevalence of hypertension, diabetes, high blood sugar, high triglyceride, and high total cholesterol between the victim and control groups. Based on guidelines of academic society in Japan $[11,12]$, we defined hypertension, diabetes, high blood sugar, high triglyceride, and high total cholesterol as follows: hypertension=systolic blood pressure $>140 \mathrm{mmHg}$ or diastolic blood pressure $>90 \mathrm{mmHg}$; diabetes $=\mathrm{HbAlc}>6.1 \%$; high blood sugar=blood sugar $>126 \mathrm{mg} / \mathrm{dL}$; high triglyceride=triglyceride $>150 \mathrm{mg} / \mathrm{dL}$; and high total cholesterol=total cholesterol $>220 \mathrm{mg} / \mathrm{dL}$. To conduct ANCOVA and logistic regression analyses, we adjusted for age and sex. The level of significance was 0.05 (two-tailed). The statistical analyses were conducted using PASW Statistics 18 for Windows.

\section{Results}

Table 1 shows comparison of demographic characteristics between victim group $(n=159)$ and control group $(n=1,048)$. As shown in Table 1 , compared with the control group, the victim group was significantly older. In addition, the victim group reported significantly higher systolic and diastolic blood pressure, and lower triglyceride and total cholesterol. The proportion of men was significantly lower in the victim (43.4\%) than in the control group (53.2\%), whereas the proportion of

Table 2: Comparison of health examination data between victim \& non-victim groups ${ }^{1)}$.

\begin{tabular}{|c|c|c|c|c|c|c|c|}
\hline & \multicolumn{3}{|c|}{ Victim group } & \multicolumn{3}{|c|}{ Non-victim group } & \multirow{2}{*}{$\mathbf{P}$} \\
\hline & $\mathbf{n}$ & Mean & $\mathrm{SE}^{2)}$ & $\mathbf{n}$ & Mean & $\mathrm{SE}^{2)}$ & \\
\hline Systolic blood pressure $(\mathrm{mmHg})$ & 158 & 136.3 & $(1.6)$ & 495 & 126.7 & $(0.9)$ & $<0.001$ \\
\hline Diastolic blood pressure $(\mathrm{mmHg})$ & 158 & 86.1 & $(1.1)$ & 495 & 81.6 & $(0.6)$ & $<0.001$ \\
\hline HbAlc (\%) & 144 & 5.1 & $(0.1)$ & 244 & 5.5 & $(0.1)$ & $<0.001$ \\
\hline Blood Sugar (mg/dL) & 158 & 108.1 & (3.5) & 583 & 119.3 & $(1.7)$ & 0.006 \\
\hline Triglyceride (mg/dL) & 157 & 143.3 & (9.9) & 660 & 178.0 & $(4.6)$ & 0.002 \\
\hline Total cholesterol (mg/dL) & 155 & 184.2 & $(3.0)$ & 718 & 205.6 & (1.3) & $<0.001$ \\
\hline
\end{tabular}

${ }^{1)}$ All analysis were conducted using analysis of covariance (ANCOVA) adjusting for sex and age

${ }^{2)}$ SE: Standard Error

Table 3: Prevalence odds ratios of clinical presentation among the victim group ${ }^{1)}$.

\begin{tabular}{|l|c|c|c|}
\hline & $\mathbf{O R}^{\mathbf{2}}$ & $\mathbf{9 5 \%} \mathbf{C l}^{\mathbf{2}}$ & $\mathbf{p}$ value \\
\hline Hypertension $^{3)}$ & 6.93 & $(4.67-10.21)$ & $<0.001$ \\
\hline Diabetes $^{3)}$ & 0.23 & $(0.10-0.54)$ & 0.001 \\
\hline high blood sugar $^{3)}$ & 0.58 & $(0.36-0.93)$ & 0.025 \\
\hline High triglyceride $^{3)}$ & 0.53 & $(0.35-0.79)$ & 0.002 \\
\hline High total cholesterol $^{3)}$ & 0.35 & $(0.22-0.55)$ & $<0.001$ \\
\hline
\end{tabular}

1) All analyses were conducted using multiple logistic regressions adjusting for sex and age. Non-victim group was reference.

2) $\mathrm{OR}=$ odds ratio; $\mathrm{Cl}=$ confidence interval.

3) Hypertension, high blood sugar, diabetes, high triglyceride, and high total cholesterol were defined as follows:

Hypertension = Systolic blood pressure $>140 \mathrm{mmHg}$ or Diastolic blood pressure $>$ $90 \mathrm{mmHg}$; Diabetes $=$ HbAlc $>6.1 \%$;

High blood sugar $=$ Blood sugar $>126 \mathrm{mg} / \mathrm{dL} ;$ High triglyceride $=$ Triglyceride $>$ $150 \mathrm{mg} / \mathrm{dL}$; High total cholesterol $=$ Total cholesterol $>220 \mathrm{mg} / \mathrm{dL}$ hypertension was significantly higher in the victim $(42.1 \%)$ than in the control group (14.5\%). The analysis revealed no other significant differences between the groups.

Table 2 shows the comparison of health examination data between the victim and control groups. After adjusting for age and sex, the victim group had significantly higher systolic and diastolic blood pressure $(F[1,648], p<0.001$ and $F[1,648], p<0.001$, respectively); lower HbA1c, blood sugar, triglyceride, and total cholesterol $(F[1,383]$, $p<0.001 ; F[1,736], p=0.006 ; F[1,813], p=0.002$; and $F[1,868], p<0.001$, respectively) compared with the control group.

Table 3 shows the results of logistic regression analyses. After adjusting for sex and age, the victim group had a significantly higher prevalence odds ratio (OR) of hypertension (OR 6.93, 95\% CI 4.67 10.21); lower prevalence ORs of diabetes, high blood sugar, high triglyceride, and high total cholesterol than control group (OR 0.23 , 95\% CI $0.10-0.54$; OR $0.58,95 \%$ CI $0.36-0.93$; OR 0.53 , 95\% CI 0.35 0.79 ; and OR $0.35,95 \%$ CI $0.22-0.55$, respectively).

\section{Discussion}

To our knowledge, this is the first study to investigate the health condition of Great East Japan Earthquake victims by using the health checks data of 159 victims three months after the event (June 2011) with 1,048 controls.

The blood pressure was significantly higher in the victim group than in the control group. The previous studies on the association of disaster with blood pressure showed that the blood pressure did not only rise during the immediate phase of disaster, but also continued to rise in the following weeks after disaster $[2-5,9,10]$. This result may be an inconsistent result. However, the finding in this study is consistent with the previous findings. Kario [2] claimed that the mechanism of hypertension after disaster is contributed by insomnia and the psychological stress resulted by the fear during disaster as well as the uncertainties that followed with environmental changes. It is pointed out that stress activates hypothalamus and sympathetic nervous system that can cause an increase of cytokine at the adrenal cortex, thus results in blood clotting as well as boost blood pressure that will cause an inflammatory reaction which may lead to the increased risk of cardiovascular disease. In fact, the death related to cardiovascular events among the elderly in the affected region was reported to increase 1.5 times in early morning and two-fold in the dawn [4]. Although the information about the history of high blood pressure was not obtained in the present study, the high prevalence of hypertension seen in the victim group (42.1\%) comparing with the control group (14.5\%) may just reviewed that the hypertension could be a major health condition in the area even before the event of disaster. On the other hand, the high prevalence of hypertension observed in the affected area maybe due to the discontinuation of medication as a result of difficulties in accessing health care. At this point, what could be assumed is that, the health management of the victims maybe still insufficient in the affected areas. In fact, the study conducted after two and a half year following the Hanshin-Awaji Earthquake on the people who lived in the temporary housing, it was shown that about $35 \%$ of the people who carried any sickness $(n=205)$ did not have sufficient treatment including medication [13]. Regarding the comparison between victom and control group, we need to consider the ecological effect to the result. In fact, the national patients' survey shows that Tohoku has higher rate of hypertension, and Tokyo has one of the lowest rate [14].

The indices of diabetes (i.e., HbA1c and blood sugar) were significantly lower in the victim group compared with the control group. 
The mean of $\mathrm{HbAlc}(5.3 \%)$ in the victim group was not different from the value showed in the National Health and Nutrition Examination Survey, 2009 [15]. Stress-induced dysfunction of the pancreas has been pointed out as relevant factors for diabetes [12], and the victims living in temporary housing and shelters are under great stress physiological and psychological stress. Foods provided in the disaster areas are often high-carbohydrate diet such as cup noodles, rice balls, and sweet bun $[7,16,17]$. Therefore, it is easy to get bored of the same content in the diet, and demotivating of food intake, which had possible caused the indicators related to diabetes less likely to rise. However, the indicators of diabetes after the earthquake is complex as multiple factors need to be considered. Therefore, further detailed investigation is needed in future.

In a similar way, the indices of hyperlipidemia (i.e., triglyceride and total cholesterol) were significantly lower in the victim group compared with the control group. We also suspect that demotivation in food intake may had caused hypoalimentation. Previous study showed that total cholesterol level has increased in government officials after the Niigata Chuetsu Earthquake [6], which is inconsistent with the present study. However, the previous data was collected after one year following the earthquake, and thus comparison is impossible because the data of the current study was collected at three months after the event. In addition, the samples were different: while administrative officers were recruited in the previous study, the present samples were local residents that are forced to live outside of their home. The different in occupational and environmental differences should be put into consideration in interpreting the results. Furthermore, as described above, it is also known that the irregular meal times and monotonous diet (noodles and high-carbohydrate diet, such as pastries and rice balls) continued a while after that earthquake [6]. According to a survey conducted in Miyagi Prefecture, which is one of the affected areas, about $90 \%$ of the shelters suffered insufficiency of calories for about two months after the earthquake [16]. In this study, based on the standard where $2,000 \mathrm{kcal}$ per day is needed for an adult, in the 239 shelter sites, calorie intake was limited to $1,500 \mathrm{kcal}$ average day, where both protein and vitamin $C$ were also below the average standard. It was pointed out that the inadequate diet issues occurred after the Great East Japan Earthquake is more serious than the Great Hanshin-Awaji Earthquake, and quite possible to have caused the decrease in total cholesterol and triglycerides [17].

\section{Limitations}

First, this study was limited only for the local residents had been living in shelters in one prefecture in the Tohoku region, also, different damages may have different effect on health conditions, therefore generalization of the result should be considered carefully. Second, although age was statistically adjusted in the study, the average age gap between the victim and control groups was 14 years, which may have influenced the results of this study. Third, as mentioned earlier, our control group was collected from the residents in Tokyo who had not lived in the shelter but experienced the consequences of disaster since we aimed to focus mainly on the effect of life in the shelter on the health condition among the victims. In a strict sense, we may have matched the controls from the same area that were not living the shelter. However, even with this approach the comparison was not made perfect because the residents living in the shelters were gathered from different areas. Furthermore, they were also influenced by another kind of stress: such as the stress directly induced by a disaster per se. To elucidate the influence of this kind of stress on the victims, the control group should be collected from (i) people from the same area before the disaster or (ii) people who live in quite far from the Tohoku region, e.g., the Kyushu region. Although the present study could not elucidate the ecological effect because the present study was not planned in advance and had some certain practical limitations, further research on effect of the earthquake per se on health condition is promising. Fourth, we understand that if possible, the demographics of the control group and the victims should match as much as possible, yet as we have no previous health data on the victims, we believed that the current control group living at home that had suffered a certain consequences of the earthquake, and their health check were assessed by the same group of people from Tokyo using the same measurements and procedures was appropriate. Fifth, we also need to note that the victim that lives in the shelter might have strong psychological distress. Previous studies show that psychological distress, including symptoms of anxiety, apathy, depression, fatigue and insomnia, increases the risk of prediabetes and Type 2 diabetes [18]. In this sense, as the participants may have exhibited symptoms of anxiety and fatigue, the results may be overestimating. Finally, considering that the other health condition (e.g., body weight, serum albumin, serum protein) and lifestyle (e.g., alcohol, tobacco, diet, and exercise) of the victims were not assessed before the earthquake, interpretation of the results should also be done with caution. In the future study, a longer term of one or two year's span should be considered, and it is necessary to sample the local residents from more areas.

\section{Conclusion}

The present study revealed that the prevalence of hypertension is higher among the victims of the Great East Japan Earthquake. The construction of medium- to long-term health management system is desired in the process of rehabilitation and reconstruction.

Moreover, in the management of victims' health when acute disaster occurs, constant blood pressure and blood test maybe necessary to confirm if the nutrition intake is efficient.

\section{Acknowledgements}

This study was conducted under a grant of the "project connecting the NPO and the affected areas," "Road Project the Foundation."

\section{References}

1. Miyagi prefecture: Related information on Great East Japan Disaster. Accessed March 18, 2013 (in Japanese)

2. Kario K (2001) Mechanism of cardiovascular events in disaster and its risks Heart 39: 110-119 (in Japanese).

3. Kobayashi S, Hirata K (2011) The management of blood pressure in a chronic phase after disaster. Blood pressure 18: 761-764 (in Japanese)

4. Kario K, Matsuo T, Shimada K, Pickering TG (2001) Factors associated with the occurrence and magnitude of earthquake-induced increases in blood pressure. Am J Med 111: 379-384.

5. Minami J, Kawano Y, Ishimitsu T, Yoshimi H, Takishita S (1997) Effect of the Hanshin-Awaji earthquake on home blood pressure in patients with essential hypertension. Am J Hypertens 10: 222-225.

6. Azuma T, Saito R, Ogawa K, Hoshino E, Nakamura E (2010) The effect of disaster on health of the municipality government officers who handle disaster response - Notice the change in the value of health diagnostic testing before and after the Niigata Chuetsu Earthquake. Niigata Medical Journal 124: 671681 (in Japanese).

7. Takeichi K (2010) Rethink the lessons learned from the disaster patient education. Practice 27:3 (in Japanese).

8. Utagawa T, Ikeda K, Muramatsu Y, Sato K (2007) Chuetsu Earthquake effects on glycemic control- aggravation factors seen from changes in the living environment Niigata Medical Journal 121: 90-96 (in Japanese).

9. Satoh M, Kikuya M, Ohkubo T, Imai Y (2011) Acute and subacute effects of the great East Japan earthquake on home blood pressure values. Hypertension 58: e193-e194. 
Citation: Kubota K, Inoue A, Shimizu Y, Kagata S, Yong R, et al. (2013) Health-Related Problems after the Great East Japan Earthquake: An Evaluation Based on the Annual Health Examination. J Nurs Care 2: 134. doi:10.4172/2167-1168.1000134

10. Konno S, Hozawa A, Munakata M (2013) Blood pressure among public employees after the great East Japan earthquake: the watari study. Am J Hypertens 26: 1059-1063.

11. The Japanese Society of Hypertension (2013) Hypertension guideline

12. The Japan Diabetes Society homepage. Accessed March 18, 2013

13. Ando S, Nakata Y, Watanabe M, Katayama K, Watanabe T, et al. (1999) Life and health condition among temporary house inhabitants who suffered from the Great Hanshin-Awaji Earthquake and evaluation of implemented continual nursing interventions. Journal of Kobe City College of Nursing 3: 29-38 (in Japanese).
14. The Ministry of Health, Labor and Welfare: Patient Survey 2008. Accessed Sep 19, 2013 (in Japanese).

15. The Ministry of Health, Labor and Welfare: National Health and Nutrition Survey 2009. Accessed March 18, 2013 (in Japanese).

16. Mainichi News dated on 2011.04.25 (in Japanese).

17. Sankei News dated on 2011.05.02 (in Japanese) .

18. Eriksson AK, Ekbom A, Granath F, Hilding A, Efendic S, et al. (2008) Psychological distress and risk of pre-diabetes and Type 2 diabetes in a prospective study of Swedish middle-aged men and women. Diabet Med 25: 834-842. 